\section{Hohe Fehlerquote bei Inhalatoren}

Melzer AC et al. Patient characteristics associated with poor inhaler technique among a cohort of patients with COPD. Resp Med 2017; 123:

$124-130$

Die Vielfalt der Inhalatoren mit unterschiedlichen Applikationsweisen und Atmungsvorgaben erschwert den korrekten Gebrauch. Die Komplexität begründet die Annahme, dass individuelle Risiken die Anwendungskompetenz beeinträchtigen könnten. Die Querschnittsstudie belegte häufige Nutzerfehler mit unterschiedlichen Ergebnissen für die Inhalator-Subtypen und differenzierte Personengruppen, bei denen besonders oft ein fehlerhafter Gebrauch bestand.

Inhalierbare Medikamente bilden die Basis der COPD-Therapie und verbessern bei richtiger Anwendung die klinischen Ergebnisse. Im Unterschied zu oralen Substanzen bedeuten sie für viele Patienten eine Herausfoderung: langsame und schnelle Inhalationsvorgaben, schütteln oder nicht schütteln, umständliche Beipackzettel. Für eine effektive Wirkung sind zwischen 10 und 14 Einzelschritte durchzuführen. Frühere Studien bezifferten die Fehlerrate auf bis zu $80 \%$. Primärer Endpunkt der Studie waren $\geq 20 \%$ Fehler im Anwendungsablauf. Die Häufigkeiten wurden für alle Inhalatoren und 3 Subtypen ermittelt:

- Dosieraerosole (MDI),

- Tiotropium-Kapsel-Inhalator,

- Diskus-Inhalator.

Die Patienten erhielten Standard-Fragebögen zu COPD-Stadium, Bildungsstand, Anamnese und soziodemografischen Faktoren sowie eine Lungenfunktionsprüfung. Die Inhalationstechnik wurde mit Dummys überprüft und standardisiert protokolliert. Insgesamt $633 \mathrm{~Pa}$ -

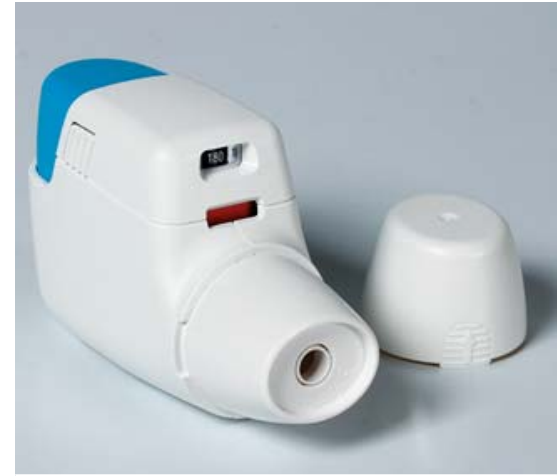

- Die Anwendung von Inhalatoren ist laut Studienergebnissen mit einer hohen Fehlerquote behaftet. Bildquelle: Michael Zimmermann/Thieme Verlagsgruppe

tienten (40-75 Jahre) mit einer COPD benutzen $\geq 1$ Inhalator und nahmen komplett an der Studie teil. 70\% waren weiße Personen, $69 \%$ hatten eine fixierte Atemwegsobstruktion und $32 \%$ rauchten. Das Geschlechterverhältnis war ausgeglichen.

$65,5 \%$ der Patienten wendeten die Inhalatoren falsch an. Besonders häufig waren Fehler bei MDI (65,7\%), gefolgt vom Diskus-Inhalator (49,5\%) und dem Tiotropium-Kapsel-Inhalator (33,9\%). Die inkorrekten Anwendungen betrafen hauptsächlich 3 Applikationsschritte:

- unvollständige Ausatmung vor der Inhalation,

- am Inhalator vorbeiströmende Luft,

- unzureichendes Luftanhalten.

Die Nutzung mehrerer Devices und die Anwendungsfehler waren nur schwach assoziiert. Wesentlichen Einfluss auf die korrekte/inkorrekte Applikation hatten die Ethnie und der Bildungsstand. Schwarze Menschen hatten ein insgesamt 3,25-fach gesteigertes Risiko für den fehlerhaften Gebrauch. Eine höhere Bildung reduzierte hingegen die Wahrscheinlichkeit. Bei den MDI und KapselInhalatoren standen ethnische Einflussfaktoren und bei den Diskus-Inhalatoren der Ausbildungsgrad im Vordergrund.

\section{FAZIT}

Die Persistenz einer starken Assoziation zwischen Ethnie, Ausbildung und Inhalationstechnik bezeichnen die Autoren als Besorgnis erregend. Ein formales Training sei eine absolute Notwendigkeit. Bei Fehlkapazitäten in der ärztlichen Praxis könnten möglicherweise Apothekenmitarbeiter diese Aufgabe übernehmen. Dies stelle allerdings eine neue, zusätzliche Barriere für die Patienten dar. Das Inhalations-Training und die Aufklärung über die Krankheit sollten Teil der Routineversorgung sein, meinen die Autoren.

Dr. med. Susanne Krome, Melle 